\title{
PERCEPÇÃO DE TÉCNICOS DE ENFERMAGEM SOBRE O USO DE EQUIPAMENTOS DE PROTEÇÃO INDIVIDUAL EM UM SERVIÇO DE URGÊNCIA ${ }^{1}$
}

\author{
PERCEPTION OF NURSING TECHNICIANS ON THE \\ IMPORTANCE OF USING PERSONAL PROTECTIVE EQUIPMENT IN \\ EMERGENCY SERVICE
}

\author{
LA PERCEPCIÓN DE LOS TÉCNICOS DE ENFERMERÍA \\ EN LA IMPORTANCIA DE UTILIZAR EQUIPO DE PROTECCIÓN \\ PERSONAL EN EL SERVICIO DE EMERGENCIA
}

\author{
Eliete Albano de Azevedo Guimarães* \\ Gustavo Dias Araújo ** \\ RiCARDO BEZERRA ${ }^{* * *}$ \\ Renata Cristina da Silveira ${ }^{* * * *}$ \\ Valéria ConCeição de OliveIra ${ }^{* * * *}$
}

\begin{abstract}
RESUMO
Introdução: $\mathrm{O}$ atendimento de urgência pré-hospitalar funciona como uma porta de entrada ao Sistema Único de Saúde (SUS). Os profissionais deste serviço, especificamente os técnicos de enfermagem, necessitam de uma maior agilidade para sobrevida do paciente. Para atender à urgência, às vezes eles se esquecem do cuidado primário ou universal, como o uso de Equipamentos de Proteção Individual (EPI). Este fato aumenta a exposição deste trabalhador ao risco ocupacional. Objetivo: compreender a percepção dos técnicos de enfermagem que atuam no Serviço de Atendimento Móvel de Urgência (SAMU) sobre a importância do uso de EPI. Método: Realizadas entrevistas a partir de um roteiro semi-estruturado com cinco técnicos de enfermagem. Foi utilizada a abordagem qualitativa do Discurso do Sujeito Coletivo - DSC para a análise dos dados, sendo possível analisar e interpretar a subjetividade dos trabalhadores estudados. Resultado: Os técnicos de enfermagem demonstraram-se inseguros na compreensão sobre os riscos biológicos ocupacionais durante o atendimento, no desconhecimento sobre as Normas Regulamentadoras 9 e 32 e sobre a utilização adequada dos EPI's no atendimento às vítimas, além do não fornecimento regular destes equipamentos pela instituição. Considerações finais: $\mathrm{O}$ desconhecimento da legislação de segurança do trabalho, dos riscos ocupacionais e da utilização adequada de EPI pode aumentar o risco de acidentes de trabalho dos técnicos de enfermagem que atuam no SAMU. Medidas promocionais são necessárias para a segurança do trabalho.
\end{abstract}

Palavras chave: Saúde do trabalhador, riscos ocupacionais, equipamentos de proteção, enfermagem.

\footnotetext{
${ }^{1}$ Trabalho extraído da monografia "Equipamentos de Proteção Individual para riscos biológicos: a compreensão ao seu uso pelos técnicos de enfermagem em um Serviço de Atendimento móvel de urgência” apresentada à Faculdade São Camilo Minas Gerais, Brasil.

*Enfermeira, docente da Universidade Federal de São João Del Rei, Minas Gerais, Brasil, Email: elietealbano@hotmail.com

** Enfermeiro do trabalho da cidade de Divinópolis/MG, Enfermeiro Socorrista do SAMU cidade de Itaúna/MG- Brasil. Email: gusenfer@hotmail.com

${ }^{* * *}$ Enfermeiro, docente da Universidade Federal de São João Del Rei, Minas Gerais, Brasil, Email: ricardocavalcanteufmg@ yahoo.com.br

${ }_{* * * *}$ Enfermeira, docente da Universidade Federal de São João Del Rei, Minas Gerais, Brasil, Email: renatacps@hotmail.com

${ }^{* * * * *}$ Enfermeira, docente da Universidade Federal de São João Del Rei, Minas Gerais, Brasil, Email: valeria.oli.enf@gmail.com
} 


\begin{abstract}
Introduction: The pre-hospital emergency situation serves as a gateway to the National Health System (SUS). Professionals from this service, specifically nursing technicians, need greater flexibility for patient survival. To respond to emergencies, sometimes they forget the primary or universal care procedures as the use of Personal Protective Equipment (PPE). This fact, increases the occupational risks among workers. Objective: To know the perception of the nursing technicians who work in the mobile emergency service (SAMU) about the importance of using personal protective equipment. Method: Semi-structured interviews with five nursing technicians were conducted. Qualitative approach of Collective Subject Discourse - DSC, which can analyze and interpret the subjectivity of the workers studied, was used for data analysis. Results: Nursing technicians have showed unawareness of biological work risks when attending urgent care, as well as ignorance of Regulating Rules 9 and 32 and correct use of personal protective equipments and no regular supply of them by the institution. Conclusions: The ignorance of work safety rules and occupational risks and misuse of personal protective equipment can increase the risk of work accident to nursing technicians working at SAMU. Promotional actions need to be taken to a safer working practice.
\end{abstract}

Key words: Occupational health, occupational risks, protective devices, nursing.

\title{
RESUMEN
}

Introducción: La situación de emergencia pré-hospitalaria sirve como puerta de entrada al Sistema Nacional de Salud (SUS). Profesionales de atención de urgencia, sobre todo técnicos de enfermería, deben trabajar muy rápido para garantizar la supervivencia del paciente. Con el fin de asistir a esta urgencia, a veces, se olvidan de los procedimientos de atención primaria o universal, como el uso correcto de los equipos de protección personal (EPP). Esto aumenta los riesgos profesionales de los trabajadores. Objetivo: Conocer la percepción de los técnicos de enfermería, que trabajan en el servicio de emergencia móvil (SAMU), sobre la importancia de utilizar equipo de protección personal. Método: Se realizaron entrevistas semiestructuradas con cinco técnicos de enfermería. Para el análisis de datos se utilizó el enfoque cualitativo del Discurso del Sujeto Colectivo - DSC, que puede analizar e interpretar la subjetividad de los trabajadores estudiados. Resultados: Los técnicos de enfermaría, en su discurso, han demostrado tener una conciencia insegura de riesgos laborales biológicos durante el servicio, la ignorancia de las Normas de regulación 9 y 32 y el uso adecuado de los EPP en el cuidado de las víctimas, y no hay suministro regular de dicho equipo por la institución. Conclusión: La ignorancia de la legislación de la seguridad en trabajo, de los riesgos laborales y el uso inapropriado de los EPP aumentan el riesgo de accidentes de técnicos de enfermería que trabajan en el SAMU. Son necesarias medidas de promoción para la seguridad en el trabajo.

Palabras clave: Salud laboral, riesgos laborales, equipos de seguridad, enfermería.

Fecha recepción: 29/11/10 Fecha aceptación: 25/10/11

\section{INTRODUÇÃO}

As causas externas configuram-se como um importante problema de Saúde Pública no Brasil e no mundo. Em muitas áreas do Brasil, já representam a segunda causa de morte, o que mostra uma tendência crescente da necessidade de um atendimento de qualidade
(1). Diante do quadro de calamidade pública o governo federal estabeleceu a Portaria Gabinete Ministerial da Saúde GM/MS No 2048 (Regulamento Técnico dos Sistemas Estaduais de Urgência e Emergência, publicado em novembro de 2002) (2) e a Portaria GM/MS No 1863 (Política Nacional de Atenção às Urgências publicada em 2003) (3), ambas pautadas em uma lógica de implantação de uma 
rede hierarquizada, regionalizada e descentralizada. Estas legislações são delineadas a partir da necessidade de organizar uma rede assistencial de atenção pré-hospitalar fixa e móvel, ligadas à atenção hospitalar (4).

O Serviço de Atendimento Móvel de Urgência (SAMU) é o principal componente da Política Nacional de Atenção às Urgências, que tem como finalidade proteger a vida das pessoas e garantir a qualidade no atendimento no Sistema Único de Saúde SUS. Seu objetivo é reduzir o número de óbitos, o tempo de internação em hospitais e as sequelas decorrentes da falta de socorro precoce (2).

A instituição do SAMU foi regulamentada pela Portaria GM/MS 1864/2003 que estabelece um veículo de suporte básico à vida para cada grupo de 100.000 a 150.000 habitantes e um avançado para 400.000 a 450.000 habitantes. O serviço é composto por Unidades de Suporte Básico (USB) e Unidades de Suporte Avançado (USA). A equipe profissional da USB é composta por motorista, auxiliar de enfermagem e/ou técnico de enfermagem. A equipe da USA é formada por médico, enfermeiro e motorista (4).

As Unidades de Suporte Básico à Vida (USB) são destinadas ao transporte inter-hospitalar de pacientes com risco de vida conhecido e ao atendimento pré-hospitalar de pacientes com risco de vida desconhecido que, no entanto, não apresentem potencial necessidade de intervenção médica no local ou durante o transporte (4).

Por ser um serviço de muita responsabilidade e cuidado, os profissionais de enfermagem e medicina desta área necessitam de educação permanente em saúde específica para o cuidado durante situações que exijam deste profissional agilidade, concentração e tranquilidade durante $o$ atendimento de urgência. Corroborando com o artigo $3^{\circ} \mathrm{da}$ GM/ MS 1863/2003 devem ser realizadas a capacitação e a educação continuadas das equipes de saúde de urgência e emergência envolvendo os profissionais de nível superior e técnico, de acordo com as diretrizes do SUS (3).

Visando a segurança do trabalhador no Brasil, há 34 Normas Regulamentadoras (NR) que são relativas à segurança e à medicina do trabalho de observância obrigatória pelas empresas privadas e públicas e pelos órgãos públicos das administrações diretas e indiretas, bem como pelos órgãos dos Poderes Legislativo e Judiciário, que possuam empregados regidos pela Consolidação das Leis do Trabalho - CLT (5). Para o trabalho desenvolvido pelos profissionais do SAMU, podemos destacar NR 6, NR 9 e a NR 32 (6).

A NR 9 estabelece a obrigatoriedade da elaboração e implementação, por parte de todos os empregadores e instituições que admitam trabalhadores como empregados, do Programa de Prevenção de Riscos Ambientais - PPRA, visando a preservação da saúde e da integridade dos trabalhadores, através da antecipação, reconhecimento, avaliação e consequente controle da ocorrência de riscos ambientais, existentes ou que venham a existir no ambiente de trabalho, tendo em consideração a proteção do meio ambiente e dos recursos naturais. Segundo esta norma, consideram-se riscos ambientais os agentes físicos, químicos e biológicos existentes nos ambientes de trabalho que, em função de sua natureza, concentração ou intensidade e tempo de exposição, são capazes de causar danos à saúde do trabalhador (7).

Nos últimos anos, a legislação trabalhista no Brasil apresentou avanços que contemplaram as questões relacionadas à segurança e à saúde dos trabalhadores da área da saúde, mesmo que de maneira inespecífica. Entretanto, como proposta positiva, foi regulamentada a normatização das condições adequadas em que o trabalho deve ser realizado, por meio da Norma Regulamentadora - 32 (NR 32), de Segurança e Saúde no Trabalho em Estabelecimentos de Assistência à Saúde (8).

A NR 32 tem por finalidade estabelecer as diretrizes básicas para a implementação de medidas de proteção à segurança e à saúde 
dos trabalhadores dos serviços de saúde, bem como daqueles que exercem atividades de promoção e assistência à saúde em geral (9).

Os profissionais de saúde podem sofrer Acidentes de Trabalho (AT) em decorrência do contato com variados agentes propiciadores de riscos ocupacionais $(10,11)$. Além disso, os profissionais deste atendimento de urgência e emergência possuem um grande risco potencial de adquirir doenças ocupacionais.

Para a NR 32 o profissional de saúde está susceptível aos riscos ocupacionais como biológicos, químicos, físicos, radiações ionizantes, dentre outros. Os materiais biológicos são responsáveis pelas infecções agudas e crônicas, causadas por vírus, fungos, bactérias, entre outros, além de serem os principais geradores de periculosidade e insalubridade aos trabalhadores.

Dentre os riscos biológicos, é comum a exposição às doenças infecciosas, como Hepatite B (HBV) e Síndrome da Imunodeficiência Adquirida (AIDS). Sabe-se, que o HBV e o HIV são transmitidos, parenteralmente, por exposição percutânea ou por mucosa, através do sangue ou de outros fluidos orgânicos em sua exposição direta (12).

Estudos mostram que os riscos de contrair a AIDS e Hepatite B estão entre os mais temidos pelos trabalhadores hospitalares, especialmente em consequência de acidentes com perfurocortantes, cujos índices de infecção têm sido estimados entre 0,25 e $0,4 \%$ para o vírus HIV, entre 6 e $30 \%$ para o HBV e entre 0,4 e $1,8 \%$ para o da Hepatite C (HBC) $(13,14)$.

Para proteger estes trabalhadores, os Equipamentos de Proteção Individual (EPI's) são recursos primordiais para prevenir a exposição aos riscos, dentre eles, os biológicos. $\mathrm{O}$ risco a este tipo de exposição, para os técnicos de enfermagem, está ligado a sua atividade desempenhada, no que se refere ao atendimento pré-hospitalar, podendo observar um risco aumentado devido à característica da assistência prestada, muitas vezes em situ- ações extremamente complexas, como o tipo de trauma e o estresse na agilidade durante o atendimento.

De acordo com a NR 6, EPI é todo dispositivo ou produto, de uso individual utilizado pelo trabalhador, destinado à proteção a riscos suscetíveis de ameaçar a segurança e a saúde no trabalho. A empresa é obrigada a fornecer aos empregados, gratuitamente, o EPI adequado ao risco, em perfeito estado de conservação e funcionamento e em quantidade que supra a necessidade de seu uso sempre que necessário pela equipe (15). Sendo assim, o uso do equipamento auxilia na prevenção de AT junto à equipe de enfermagem.

Acidente de trabalho (AT) é aquele que ocorre pelo exercício do trabalho a serviço da empresa ou pelo exercício do trabalho do segurado. Pode causar desde um simples afastamento até a perda, redução da capacidade laborativa ou até mesmo a morte do segurado. O AT típico ocorre durante o desempenho laboral e o AT de trajeto acontece durante o deslocamento entre a residência e o local de trabalho (16). Considera-se AT a doença profissional e a doença do trabalho. Equiparam-se também ao AT: o acidente ligado ao trabalho que, embora não tenha sido a causa única, haja contribuído diretamente para a ocorrência da lesão; certos acidentes sofridos pelo segurado no local e no horário de trabalho; a doença proveniente de contaminação acidental do empregado no exercício de sua atividade; e o acidente sofrido a serviço da empresa ou no trajeto entre a residência e o local de trabalho e vice-versa (17).

No entanto, os AT podem ser evitáveis e causam um grande impacto sobre a produtividade e a economia, além de grande sofrimento social, tendo os seus custos raramente contabilizados, pois, muitas vezes acometem trabalhadores sem registro formal em carteira de trabalho. Sabe-se que trabalhadores que atuam nos serviços de saúde têm possibilidades de adquirir enfermidades e sofrer AT em decorrência do contato com situações 
de riscos ocupacionais variadas, pela multiplicidade de fatores de risco biológico, físico, psíquico e ergonômicos.

As precauções durante o atendimento incluem a utilização de barreiras para proteção, como o uso de EPI. Utilizá-los corretamente é de suma importância, pois permite a realização de procedimentos de forma segura, tanto para o profissional que está prestando assistência quanto ao paciente que está sendo assistido (18).

Neste sentido o objetivo do estudo foi o de compreender a percepção dos técnicos de enfermagem que atuam no SAMU sobre o uso de EPI.

\section{METODOLOGIA}

Estudo de abordagem qualitativa desenvolvido em Itaúna, Minas Gerais, em 2009. Itaúna encontra-se situada na Macrorregional Oeste do Estado, região composta por 57 municípios, perfazendo 06 Microrregiões, sendo uma das sedes microrregionais de saúde. Sua população estimada em 85.838 habitantes encontra-se distribuída em 66 bairros na zona urbana e 38 comunidades rurais (19).

Em concordância com o Termo de Compromisso de Gestão entre as instâncias de governo, o município assumiu responsabilidades como a ampliação da rede de saúde, com ênfase na estruturação da atenção primária em saúde, na organização de serviços de vigilância em saúde, serviços especializados e de urgência, sendo também referência na pactuação de ações integradas entre as microrregionais de saúde.

O SAMU, um dos serviços de urgência implantado em Itaúna, possui um total de oito técnicos de enfermagem que trabalham em escala de 12 horas por 72 horas de folga na Unidade de Suporte Básico de Vida (USB). A equipe completa possui, por escala, dois profissionais, sendo um motorista e um técnico de enfermagem.
Foram realizadas entrevistas, a partir de um roteiro (em anexo), com os técnicos de enfermagem. Neste roteiro havia cinco questões norteadoras da entrevista que abordavam as seguintes temáticas: o conhecimento dos sujeitos sobre os riscos biológicos a que estavam expostos durante o processo de trabalho; o conhecimento dos sujeitos sobre NR 6 e 32; os EPI's utilizados durante os atendimentos; as dificuldades encontradas para a utilização e aquisição de EPI's.

Durante as entrevistas, os sujeitos tiveram liberdade para abordar o assunto sob o seu ponto de vista, permitindo tanto a obtenção de informações objetivas quanto as subjetivas, referentes às atitudes, opiniões e valores de sua subjetividade (20).

As entrevistas foram audiogravadas e realizadas no período de julho a setembro de 2009. Todos os técnicos de enfermagem foram convidados a participar de uma entrevista na USB, que era agendada previamente e obedeceu aos princípios éticos contidos na Resolução No 196/96, do Conselho Nacional de Saúde do Ministério da Saúde Brasileiro.

A equipe, ao todo, era compreendida por oito técnicos de enfermagem. Destes, cinco consentiram em participar da pesquisa e assinaram o Termo de Consentimento Livre e Esclarecido (TCLE).

A análise e a organização dos dados foram desenvolvidas a partir da abordagem qualitativa do Discurso do Sujeito Coletivo - DSC (21), sendo possível analisar e interpretar a subjetividade dos trabalhadores estudados.

O DSC é uma síntese de trechos de vários discursos com sentidos semelhantes. A seguir, os discursos semelhantes foram reunidos num só discurso. O DSC fundamenta-se a partir da teoria da Representação Social e seus pressupostos sociológicos. A técnica do DSC consiste em analisar o material verbal coletado em pesquisas que tenham depoimentos como sua matéria prima, extraindo-se de cada um as "idéias centrais ou ancoragens" e as suas correspondentes "expressões chave". Com as "ideias centrais/ancoragens 
e expressões chave" semelhantes compõe-se um ou vários discursos-síntese que são os DSC (21).

Neste sentido, para análise, o discurso-síntese foi construído a partir da reconstrução do discurso pessoal subjetivo, tendo como proposta visualizar um melhor entendimento sobre o uso dos EPI pelos trabalhadores em foco. Cada discurso dos entrevistados foi identificado com a sigla DSC, seguido de uma numeração de 1 a 5 .

Este estudo foi aprovado pelo Comitê de Ética em Pesquisa da Universidade do Estado de Minas Gerais, Brasil, através do parecer No $76 / 2009$.

\section{RESULTADOS E DISCUSSÃO}

Durante toda a pesquisa buscou-se considerar os aspectos pertinentes para construção de uma análise sobre a percepção dos técnicos de enfermagem que atuavam no SAMU, sobre a importância do uso dos EPI durante a assistência de enfermagem prestada.

Para interpretação dos discursos produzidos pelos técnicos de enfermagem, recorreu-se à literatura, por meio de teorias explicativas, que serviram para contextualizar, analiticamente, os temas centrais que emergiram durante as entrevistas, sendo estes categorizados como: "A compreensão sobre os riscos biológicos, durante o atendimento de emergência", "O conhecimento sobre as Normas Regulamentadoras NR 6 e NR 32, bem como sua importância" e "A utilização de Equipamentos de Proteção Individual (EPI) e seu fornecimento pela Instituição".

\section{A compreensão sobre os riscos biológicos, durante $o$ atendimento de emergência}

Em relação a esta categoria, verificou-se nos discursos, a preocupação dos trabalhadores com apenas algumas possibilidades de contaminação. No entanto, não relataram sobre patologias decorrentes da contaminação e outras possibilidades que se constituiriam risco para a própria saúde.

"O que me lembro é o sangue, secreção, pérfuros, produtos químicos, fluídos corporais e várias doenças como Hepatite e HIV" (DSC1).

No ambiente profissional há multiplicidade de riscos aos trabalhadores de enfermagem: os biológicos, físicos, químicos e ergonômicos. Os biológicos são os responsáveis por infecções agudas e crônicas, ocasionadas por vírus, fungos e bactérias. Os físicos são causados pelas radiações, vibrações, ruídos, temperatura ambiental, iluminação e eletricidade. Os riscos químicos são os gerados pelo manuseio de uma variedade de substâncias químicas e também pela administração de medicamentos que podem provocar desde simples alergias até importantes neoplasias. Os ergonômicos são gerados principalmente pela postura irregular dos profissionais de enfermagem em situações como movimentação de pacientes, flexões de coluna frequentes, entre outros (22).

O conhecimento das doenças e suas particularidades, assim como o risco que o trabalhador de enfermagem possui durante o exercício de sua profissão, auxiliam o profissional em possíveis condutas de prevenção e cuidado, o que pode diminuir os AT durante $\mathrm{o}$ atendimento.

\section{O conhecimento e a importância das Normas Regulamentadoras No 6 e No 32}

A Norma Regulamentadora 32 é considerada de extrema importância no cenário brasileiro, como legislação federal específica que trata das questões de segurança e saúde no trabalho, no setor da saúde. É a normatização de referência, pois outras normatizações existentes encontram-se esparsas, reunidas em diversas outras NRs e resoluções, que não foram construídas especificamente para as necessidades dos serviços de saúde (23). 
Acredita-se que mudanças benéficas poderão ser alcançadas por meio da referida normatização, uma vez que procedimentos e medidas protetoras deverão ser realizados como forma de promover segurança no trabalho e prevenir AT.

A NR 6 determina a utilização dos Equipamentos de Proteção Individual (EPI) pelos profissionais, bem como o seu fornecimento pelas instituições. Porém, evidências científicas têm mostrado que trabalhadores da saúde submetem-se aos vários agentes de riscos ocupacionais em seus ambientes de trabalho e nem sempre utilizam os EPI's normatizados pela NR 6 (24).

As NR's complementam-se para desencadear a prevenção do risco ocupacional, ao profissional de enfermagem que está exposto durante as suas atividades assistenciais. No entanto, este estudo revelou, por meio dos discursos, que há um desconhecimento e desinteresse por parte dos sujeitos em aplicar as normas regulamentadoras.

"Nunca ouvi, deve ser uma novidade? O pessoal da enfermagem não se preocupa com isso e se conhece a NR 32, maior problema é colocar em prática" (DSC 2).

"Não iremos ler a NR 32 porque estamos sem tempo e também não serve de nada para os técnicos de enfermagem" (DSC 3).

"Não ouvi falar desta NR 6 e se ouvi não entendi nada"( DSC 6).

É importante destacar que os discursos relatados anteriormente demonstram ainda duas situações que necessitam reflexões. Primeiramente, a leitura das entrelinhas dos discursos demonstrou que os sujeitos vêem na técnica a grande relevância do seu processo de trabalho. Assim, normas, legislações, aspectos teóricos da prática de enfermagem, para estes entrevistados, possuem pouca importância, não sendo valorizados e muito menos incorporados ao seu processo de trabalho. Isto certamente, já confere um fator de risco para contaminação, bem como para acidentes. A falta de informação e de reflexão é um fator desencadeante para a não aderência dos profissionais ao uso dos EPI, potencializando assim os riscos e AT $(25,26)$.

Outra situação em destaque é a presença de alguns fatores que podem estar limitando a aderência ao uso dos EPI, como a característica do processo de trabalho. Sabe-se que, no SAMU as atividades são desenvolvidas em meio a um processo norteado pela necessidade de agilidade, dinamicidade e que, muitas vezes, ocorrem em situações tensas (27). No entanto, estas características não devem impedir a reflexão sobre as práticas, sobre o paciente assistido, sobre os procedimentos e sobre as possibilidades de prevenção, bem como sobre a necessidade de utilização das barreiras de proteção do próprio profissional. Este estudo revelou que os profissionais necessitam ser inseridos num processo informacional, de educação em saúde e incentivo à prevenção durante o processo de trabalho.

Acredita-se que mudanças benéficas poderão ser alcançadas por meio da normatização, uma vez que procedimentos e medidas protetoras deverão ser utilizados com vistas a promover segurança no trabalho e prevenção de acidentes e doenças ocupacionais. A partir do momento em que este profissional desconhece essas normas, ou se conhece não as aplica, confirma-se o risco de acidentes, bem como de contaminações.

\section{A utilização de Equipamentos de Proteção Individual (EPI) e seu fornecimento pela instituição}

Os profissionais da saúde são potencialmente vulneráveis à exposição aos agentes microbiológicos, devido ao contato direto e constante com o paciente, aumentando o risco de contrair uma doença ocupacional. Assim, faz-se necessária a utilização de barreiras para proteção, conferidas pelo uso de EPI.

Conforme a Lei Brasileira n. 2048 (2), o uso de EPI preconizados no serviço de atendimento móvel de urgência são: luvas de 
procedimento, óculos de proteção, máscara do tipo cirúrgica, macacão e botas, que são usados durante as atividades de contato direto com o paciente.

No estudo, foi constatado, a partir das falas dos entrevistados, somente o uso de luvas de procedimento e macacão fornecidos pela instituição.

"Durante o atendimento utilizamos somente luvas de procedimento, macacão e botas" (DSC 4).

Desta forma, percebe-se que os óculos de proteção não são citados como EPI utilizados nas atividades do serviço. Estes equipamentos seriam a barreira de proteção da via ocular, que neste caso, está exposta aos vários riscos de contaminação. É muito comum, no serviço de urgência, o contato com secreções, fluidos e dejetos que podem ser projetados contra o profissional de enfermagem em assistência ao paciente. Então fica clara a exposição ao risco diante da ausência dos óculos de proteção.

Além da insuficiente utilização dos EPI, grande parte dos sujeitos relatou um fornecimento inadequado dos equipamentos pela instituição que, por lei, é obrigatório.

"A instituição só fornece aos técnicos de enfermagem luvas de procedimento" (DSC 5).

"Não tenho nenhuma restrição ao uso do EPI o problema é seu fornecimento pelo serviço" (DSC 7).

Segundo o artigo 6.3 da NR 6 os empregadores ficam obrigados a fornecer aos seus empregados, de forma gratuita, o EPI adequado ao risco característico do setor onde presta seus serviços. Além do fornecimento dos EPI's pela empresa, os mesmos devem estar em perfeitas condições de uso (6). Não é o que ocorreu na instituição em estudo. Isto ficou claro nos discursos analisados. Este descumprimento da regulamentação por parte da instituição pode contribuir para os
AT, e consequentemente para o afastamento do profissional, gerando encargos para a própria instituição, bem como complicações sobre o processo de trabalho do serviço móvel de urgência.

\section{CONSIDERAÇÕES FINAIS}

Os riscos nos serviços de saúde existem em virtude das inúmeras áreas de insalubridade com graduação variável, sendo influenciada a partir da complexidade do tipo de atendimento prestado, assim como, da função do trabalhador de enfermagem.

Toda a equipe de enfermagem está sujeita a AT. Porém, os técnicos e auxiliares de Enfermagem, por estarem em contato direto com o paciente, realizando procedimentos invasivos, preparando medicações, administrando medicamentos, realizando curativos e outros procedimentos que os mantêm sempre em constante contato com material biológico têm maior chance de ocorrência de AT. É relevante ressaltar que a ocorrência de AT não está relacionada apenas ao nível de formação, mas também ao treinamento, capacitação, recursos materiais disponíveis, assim como à cultura local.

Neste estudo, os resultados demonstram que estes profissionais desconhecem a legislação vigente de saúde em sua segurança ocupacional e, consequentemente, os riscos biológicos ocupacionais a que estão expostos pela não utilização adequada de EPI.

O desconhecimento, o desinteresse e o não fornecimento adequado destes EPI, necessários para o desenvolvimento de sua profissão, aumentam o risco de AT e, principalmente ao absenteísmo destes trabalhadores de enfermagem por acidentarem-se ou adquirirem uma doença ocupacional durante $o$ atendimento.

Cabe ressaltar, quanto ao fornecimento do EPI, que a instituição é obrigada a fornecer aos empregados, gratuitamente, o EPI 
adequado ao risco apresentado e em perfeito estado de conservação e funcionamento.

Baseados nas entrevistas realizadas, reitera-se a importância do uso dos EPI, pelos profissionais de enfermagem, dando-se ênfase aos esclarecimentos às questões de acidentes com material biológico e às precauções padrão, bem como o uso dos EPI's na tentativa de prevenção de AT e/ou doenças ocupacionais e, fundamentalmente, o conhecimento das leis vigentes e o cumprimento das mesmas durante o exercício profissional.

Torna-se um desafio, para todos os envolvidos neste cenário, adotar medidas que visem a mudanças de comportamento e à ampliação de estratégias para uma prática segura de trabalho.

Acredita-se que existam alguns elementos para a prevenção de acidentes junto a esses trabalhadores como o envolvimento de toda a organização, a cultura de segurança, notificação de acidentes com o preenchimento da Comunicação de Acidente de Trabalho (CAT) pelo profissional de saúde, plano de prevenção baseado em dados locais, mapa de riscos elaborados por profissionais da Saúde do Trabalhador e afixados corretamente no local de trabalho (ambulâncias, por exemplo), educação dos profissionais e avaliação das intervenções para prevenção.

Portanto, o enfermeiro e a equipe de enfermagem devem ter capacidade de ação multidisciplinar e possuir conhecimentos para que aproveitem as ferramentas disponíveis nesta área de atuação a fim de identificar os agravos e minimizar os danos causados pelo trabalho (28) e evitar possíveis adoecimentos e AT.

Dessa maneira, poder-se-á reforçar a mudança de atitude destes profissionais de saúde tão imprescindíveis para um atendimento de qualidade do SAMU, aliado a isso, este mesmo trabalhador deve estar atento quanto à adoção de melhores comportamentos preventivos e seguros, além de reivindicações que contribuam para uma melhor saúde ocupacional.

\section{REFERÊNCIAS}

1. Ministério da Saúde. Saúde Brasil 2005: uma análise da situação de saúde. Brasília, DF: Ministério da Saúde; 2005. 822 p.

2. Ministério da Saúde. Portaria no 2048, de 5 de novembro de 2002. Política Nacional de Atenção às Urgências. Brasília (Brasil): Ministério da Saúde; 2002.

3. Portaria no 1863 , de 29 de setembro de 2003. Política Nacional de Atenção às Urgências. Brasília (Brasil): Ministério da Saúde; 2003.

4. Ministério da Saúde. Manual de Regulação Médica de Urgências. Brasília, DF: Ministério da Saúde; 2006. 126p.

5. Ministério do Trabalho. Decreto-Lei ${ }^{\circ}$ 5.452, de 1 de maio de 1943. Consolidação das Leis Trabalhista-CLT (Brasil). [Internet] Brasília: Ministério do Trabalho; 1943. [citado setembro 2010]. Disponível em http://www.planalto.gov.br/ ccivil/decreto-lei/del5452.htm

6. Ministério do trabalho (BR). Manuais de legislação: Normas Regulamentadoras. São Paulo: Atlas; 2008.

7. Normas Regulamentadoras. 1994. NR 9 - Programa de prevenção de riscos ambientais. [Internet] Brasília: Ministério do Trabalho e Emprego; 1994 [citado setembro 2010]. Disponível em http:// www.mte.gov.br/legislacao/normas_regulamentadoras/nr_09_at.pdf.

8. Robazzi MLCC, Barros Junior JC. Proposta brasileira de normatização para os trabalhadores da saúde. Cienc enferm. 2005; 11(2): 11-5.

9. Normas Regulamentadoras. 2008 NR 32 - Segurança e saúde no trabalho em serviços de saúde. [Internet] Brasília: Ministério do Trabalho e Emprego; 2008 [citado setembro 2010]. Disponível em: http://www.mte.gov.br/legislacao/normas_regulamentadoras/nr_32.pdf

10. Rezende MP. Agravos à saúde de auxiliares de enfermagem resultantes da ex- 
posição ocupacional aos riscos físicos Ribeirão Preto. [Tesis Maestria]. [Ribeirão Preto (SP)]: Universidade São Pablo, 2003.

11. Murofuse NT. O adoecimento dos trabalhadores de enfermagem da Fundação hospitalar do Estado de Minas Gerais: reflexo das mudanças no mundo do trabalho. [Tese Doutorado]. [Ribeirão Preto (SP)]: Universidade São Pablo; 2004.

12. Teixeira P, Vale S. Biossegurança: uma abordagem multidisciplinar. Rio de Janeiro: Fiocruz; 1998.

13. International Healthcare Worker Safety Center. Risk of infection following a single $\mathrm{HIV}, \mathrm{HBV}$, or HCV-contaminated needlestick or sharp instrument injury. [Internet] Virginia; 2001 [citado 30 agosto 2010]. Disponível em: http:// www.healthsystem.virginia.edu/internet/epinet/estimates.cfm

14. Fonseca MMR. Profilaxia pós-exposição a material biológico para profissionais de saúde. En: Melo HRL, Brito CAA, Niranda DB Filho, Souza SG, Henriques APC, Silva OB. Condutas em doenças infecciosas. Rio de Janeiro: Medsi; 2004. p. 758-65.

15. Brasil. Ministério do Trabalho em Emprego. NR 6 - Equipamento de proteção individual - EPI. [Internet] Brasil: Ministério do Trabalho em Emprego; 2001 [citado outubro 2010)]. Disponível em: http://www.mte.gov.br/legislacao/normas_regulamentadoras/nr_06.pdf

16. Presidência da República do Brasil. Casa Civil. Subchefia para Assuntos Jurídicos. Lei no 8213 de 24 de julho de 1991. Dispõe sobre os Planos de Benefícios da Previdência Social e dá outras providências. Diário Oficial da União [Internet] Brasília; jul. 1991 [citado 24 outubro 2011]. Disponível em: http://www.planalto.gov. br/ccivil_03/leis/L8213cons.htm

17. Ministério da Assistência e Previdência Social. Anuário Estatístico da Previdência Social 2007. Seção IV - Acidentes do Trabalho. [Internet] 2011 [citado 19 outubro 2011]. Disponível em: http:// www.mpas.gov.br/conteudoDinamico. php?id=559

18. Ministério da Saúde. Manual de condutas: exposição ocupacional a material biológico: Hepatite e HIV. Secretaria de Políticas de Saúde. Coordenação Nacional de DST e Aids. Brasília: Ministério da Saúde; 2000.

19. Instituto Brasileiro de Geografia e Estatística. IBGE, Brasília (Brasil); 2000.

20. Minayo MCS. O Desafio do Conhecimento: Pesquisa Qualitativa em Saúde. Hucitec: São Paulo; 2004.

21. Lefévre F, Lefévre AMCO. Discurso do Sujeito Coletivo: um novo enfoque em pesquisa qualitativa (desdobramentos). Educs: São Paulo; 2003.

22. Bulhões I. Riscos do trabalho de enfermagem. 2a ed. Folha Carioca: Rio de Janeiro; 1994.

23. Robazzi MLCC, Marziale MHP. A Norma regulamentadora 32 e suas implicações sobre os trabalhadores de enfermagem. Rev Lat Am Enfermagem. 2004; 20(5): 834-6.

24. Suazo SVV. Contribuição ao estudo sobre acidentes de trabalho que acometem as trabalhadoras de enfermagem em hospitais chilenos. [Tese Doutorado]. [Ribeirão Preto (SP)]: Universidade São Pablo, 1999.

25. Jansen AC, Robazzi, MLCC. Accidentes de trabajo en enfermería y su relación con la instrucción recibida. Cienc Enferm. 2009; 15 (1): 49-59.

26. Oliveira AC, Gonçalves JA. Acidente ocupacional por material perfurocortante entre profissionais de saúde de um Centro Cirúrgico. Rev Esc Enferm USP. 2010; 44(2): 482-7.

27. Campos RM, Farias GM, Ramos CS. Satisfação profissional da equipe de enfermagem do SAMU/Natal. Rev. Eletrônica Enferm [Internet]. 2009 [citado outubro 2010]; 11(3): 647-57. Disponível em: 
http://www.fen.ufg.br/revista/v11/n3/ v1ln3a24.htm.

28. Ribeiro EIG, Shimizu HE. Acidentes de trabalho com trabalhadores de enfermagem. Rev Bras Enferm. 2007; 60(5): 53540.

\section{Anexo - Roteiro de Entrevista}

\section{DADOS DE IDENTIFICAÇÃO}

MUNICÍPIO:

MACRORREGIÃO:

GERÊNCIA REGIONAL DE SAÚDE:

PORTE POPULACIONAL:

ENTREVISTADO (iniciais do nome):

SEXO: FORMAÇÃO:

CARGO:

VÍNCULO PROFISSIONAL:

TEMPO DE TABALHO:

DATA DA ENTREVISTA:

/.......... /

INÍCIO:

TÉRMINO:

\section{TEMÁTICAS:}

1 - O conhecimento dos sujeitos sobre os riscos biológicos a que estavam expostos durante o processo de trabalho

Fale sobre os riscos biológicos que estão expostos (a) durante o seu atendimento?

2 - O conhecimento dos sujeitos sobre as Normas Regulamentadoras NR 6 e 32.

Você conhece a normalização sobre a segurança e saúde no trabalho em estabelecimentos de assistência à saúde? Comente sobre ela.

3 - Os EPI's utilizados durante os atendimentos.

Quais os EPI's utilizados durante seu atendimento? Cite-os.

Você conhece a normalização sobre equipamento de proteção individual? Comente sobre ela.

4 - As dificuldades encontradas para a utilização e aquisição de EPI's.

Qual a dificuldade encontrada na utilização e aquisição destes EPI’s? 